05

\title{
Магнитные свойства наночастиц NiO: вклады антиферромагнитной и ферромагнитной подсистем в различных диапазонах магнитных полей до $250 \mathrm{kOe}$
}

\author{
() Д.А. Балаев ${ }^{1,2}$, А.А. Дубровский ${ }^{1}$, А.А. Красиков ${ }^{1}$, С.И. Попков ${ }^{1,2}$, А.Д. Балаев ${ }^{1}$, \\ К.А. Шайхутдинов ${ }^{1}$, В.Л. Кириллов ${ }^{3}$, О.Н. Мартьянов ${ }^{3}$ \\ ${ }^{1}$ Институт фризики им. Л.В. Киренского ФИЦ КНЦ СО РАН, \\ Красноярск, Россия \\ ${ }^{2}$ Сибирский фредеральный университет, \\ Красноярск, Россия \\ ${ }^{3}$ Институт катализа им. Г.К. Борескова СО РАН, \\ Новосибирск, Россия \\ E-mail: dabalaev@iph.krasn.ru
}

(Поступила в Редакцию 14 фревраля 2017 г.)

Исследованы магнитные свойства наночастиц антиферромагнитного $\mathrm{NiO}$, полученного термическим разложением гидроксокарбоната никеля. Измерения намагничивания в полях до $250 \mathrm{kOe}$ показали линейный рост магнитного момента в области больших полей, обусловленный вкладом антиферромагнитно упорядоченного „ядра“ наночастиц, причем величина антиферромагнитной восприимчивости соответствует таковой для „объемного“ поликристаллического $\mathrm{NiO}$. Это дало возможность определить количественно антиферромагнитный и ферромагнитный вклады в общий магнитный отклик образца. Последний возникает благодаря неполной компенсации спинов в антиферромагнитной наночастице вследствие наличия дефектов на ее поверхности. Показано, что для корректного определения суперпарамагнитной температуры блокировки необходим учет поведения антиферромагнитной восприимчивости „ядра“ частиц.

Исследование выполнено при финансовой поддержке Российского фонда фундаментальных исследований, Правительства Красноярского края, Красноярского краевого фонда поддержки научной и научно-технической деятельности в рамках научного проекта № 17-42-240138.

DOI: $10.21883 /$ FTT.2017.08.44752.39

\section{1. Введение}

Наночастицы материалов, проявляющих антиферромагнитное (AF) упорядочение, активно исследуются в настоящее время с точки зрения фундаментальных причин изменения магнитных свойств, происходящих из-за влияния размерных и поверхностных эффектов, а также дефектов и структурных искажений [1-12]. Пожалуй, основным отличием магнитных свойств наноразмерных AF-частиц от объемных аналогов можно считать появление нескомпенсированного магнитного момента $\mu_{\text {unc }}$ в частицах малых размеров $[1,3-8,10-12]$. В результате в AF-наночастице, остающейся химически и структурно однородной, присутствуют как минимум две магнитных фазы: антиферромагнитная, присущая „ядру“ частицы, и ферромагнитная (FM), вызванная появлением $\mu_{\text {unc. На- }}$ личие магнитного момента у AF-наночастиц открывает перспективы их практического применения [13-15].

Для анализа магнитных свойств AF-наночастиц используются подходы, общепринятые для описания ферри- и ферромагнитных частиц: различаются температурные диапазоны заблокированного $\left(T<T_{\mathrm{B}}\right.$, где $T_{\mathrm{B}}-$ температура блокировки) и разблокированного $\left(T>T_{\mathrm{B}}\right)$ состояний, проявляющиеся соответственно в наличии и отсутствии магнитного гистерезиса. Значение $T_{\mathrm{B}}$ опреде- ляется известным выражением

$$
T_{\mathrm{B}}=K V / \ln \left(\tau / \tau_{0}\right) k_{\mathrm{B}},
$$

в котором $K-$ константа магнитной анизотропии, $V-$ объем частицы, $k_{\mathrm{B}}$ - константа Больцмана, $\tau$ и $\tau_{0}-$ характерные времена измерения и релаксации магнитного момента частицы. При этом $\tau_{0} \approx 10^{-9}-10^{-11} \mathrm{~s}$, а для квазистатических магнитных измерений $\tau \approx 10^{1}-10^{2} \mathrm{~s}$, что приводит к соотношению $T_{\mathrm{B}} \approx K V / 25 k_{\mathrm{B}}$.

Магнитный отклик $M_{\text {tot }}(H, T)$ AF-частиц может в первом приближении быть описан суперпозицией двух вкладов [1], соответствующих FM- и AF-фазам

$$
M_{\mathrm{tot}}(H, T)=M_{\mathrm{FM}}(H, T)+M_{\mathrm{AF}}(H, T) .
$$

При $T>T_{\mathrm{B}}$ FM-вклад моделируется функцией Ланжевена с учетом распределения по размерам или магнитным моментам частиц, при $T<T_{\mathrm{B}}$ магнитный гистерезис от FM-вклада определяется конкуренцией энергии магнитной анизотропии $K V$ и зеемановской энергией $\boldsymbol{\mu}_{\text {unc }} \mathbf{H}$. Магнитополевая зависимость AF-вклада записывается в виде: $M_{\mathrm{AF}}(H)=\chi_{\mathrm{AF}} H$, где $\chi_{\mathrm{AF}}-\mathrm{AF}-$ восприимчивость, соответствующая AF-,ядру“ частиц. При таком подходе величина $\chi_{\mathrm{AF}}$ должна быть сопоставима с соответствующим значением AF-восприимчивости объемного мате-

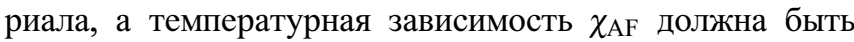


аналогичной поведению магнитной восприимчивости объемного антиферромагнетика (как в случае поликристалла со случайной кристаллографической ориентацией кристаллитов).

C другой стороны, в малых AF-частицах возможен дополнительный рост AF-восприимчивости, предсказанный Неелем [16-18] и называемый термином суперантиферромагнетизм. Этот размерный эффект заключается в том, что в AF-частицах с четным числом ферромагнитных плоскостей поверхностные спины под действием приложенного перпендикулярно оси легкого намагничивания поля $\mathbf{H}$ подворачиваются в большей степени, чем спины „внутренних плоскостей“. Заметное увеличение AF-восприимчивости может наблюдаться для частиц, имеющих в диаметре не более нескольких десятков ферромагнитно упорядоченных плоскостей. Однако при уменьшении размеров АF-наночастиц возрастающую роль играет их нескомпенсированный

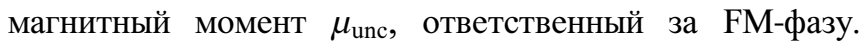
Величина $\mu_{\mathrm{unc}}$, согласно Неелю [18], определяется соотношением

$$
\mu_{\mathrm{unc}} \propto J N^{b}
$$

в котором $N$ - число магнитоактивных атомов в частице, $J$ - магнитный момент атома, а показатель степени $b$ может принимать значения в диапазоне $\sim 1 / 3-2 / 3$ в зависимости от характера дефектов в частице (для дефектов на поверхности частиц $b=1 / 3)$. Разделение $\mathrm{AF}-$ и FM-вкладов по результатам магнитных измерений малых AF-частиц представляет собой нетривиальную задачу. Дело в том, что в используемом обычно диапазоне магнитных полей до $\sim 60-90 \mathrm{kOe}$ вклад от малых по величине магнитных моментов может быть еще далек от насыщения, и это затрудняет однозначное выделение АF-вклада из кривой намагничивания. В этом случае целесообразным является использование сильных импульсных магнитных полей, что существенно расширяет диапазон измерений кривых намагничивания.

В работе [19] было показано, что „аномальное“ поведение зависимости $\chi_{\mathrm{AF}}(T)$ антиферромагнитно упорядоченного ферритина, а именно уменьшение $\chi_{\mathrm{AF}} \mathrm{c}$ ростом $T$, получаемое многими авторами [1,6,20-23] из обработки зависимостей $M(H)$ в диапазоне до $60 \mathrm{kOe}$, скорее всего является артефактом. В достаточно больших полях $(\sim 200 \mathrm{kOe})$ значения $\chi_{\mathrm{AF}}$, получаемые по производной $d M / d H$, заметно меньше, в то время как температурная зависимость $\chi_{\mathrm{AF}}(T)$ (при $H>200 \mathrm{kOe}$ ) уже ведет себя „классическим“ образом - возрастает с повышением температуры. Среди различных AFматериалов в виде наночастиц исследования в сильных импульсных полях проводились только для образцов ферритина $[18,24,25]$ и манганита лантана [26]. Ввиду этого, целесообразно исследовать и другие AFнаночастицы с помощью данной методики. В данной работе приводятся результаты исследования магнитных свойств наночастиц оксида никеля, причем в качестве первого шага был взят образец с относительно „крупными“ частицами со средним размером $\sim 25 \mathrm{~nm}$, в которых эффект суперантиферромагнетизма должен быть незначительным.

\section{2. Эксперимент}

2.1. Получение и характеризация образцов. Наночастицы $\mathrm{NiO}$ были получены методом термического разложения гидроксокарбоната никеля $\mathrm{NiCO}_{3} \cdot 0.18 \mathrm{Ni}(\mathrm{OH})_{2} \cdot 0.50 \mathrm{H}_{2} \mathrm{O}$ (никель углекислый основной). Режим нагревания: $14 \mathrm{~h}$ при $90^{\circ} \mathrm{C}$, затем в течении $5 \mathrm{~h}$ подъем температуры до $500^{\circ} \mathrm{C}$ и выдержка в течении $1 \mathrm{~h}$ при $500^{\circ} \mathrm{C}$. Этот образец обозначен как nano $\mathrm{NiO}$.

Дифракционная картина для образца nano $\mathrm{NiO}$ была получена на приборе Bruker D8 Advance (Bruker, Германия) с использованием $\mathrm{Cu} K_{\alpha}$-излучения $(\lambda=1.5418 \AA)$. Результаты представлены на рис. 1. Все наблюдаемые дифракционные пики принадлежат фазе оксида никеля $\mathrm{NiO}$ (PDF № 047-1049). Параметр элементарной кубической ячейки $\mathrm{NiO}$ совпадает со стандартным значением (пространственная группа $F m \overline{3} m, a=b=c=4.176 \AA$, $\alpha=\beta=\gamma=90^{\circ}$ ). Размер области когерентного рассеяния, определенный по уширению дифракционных пиков, составляет около $30 \mathrm{~nm}$.

Микрофотографии частиц образца nano $\mathrm{NiO}$ получены c помощью просвечивающей электронной микроскопии высокого разрешения (High-resolution transmission electron microscopy - HRTEM) на микроскопе JEOL JEM-2010 с разрешением $1.4 \AA$, работающем при ускоряющем напряжении $200 \mathrm{kV}$. На рис. 2 приведены типичные данные HRTEM, полученные на образце nano NiO. Средний размер наночастиц $\mathrm{NiO}$ (по данным нескольких

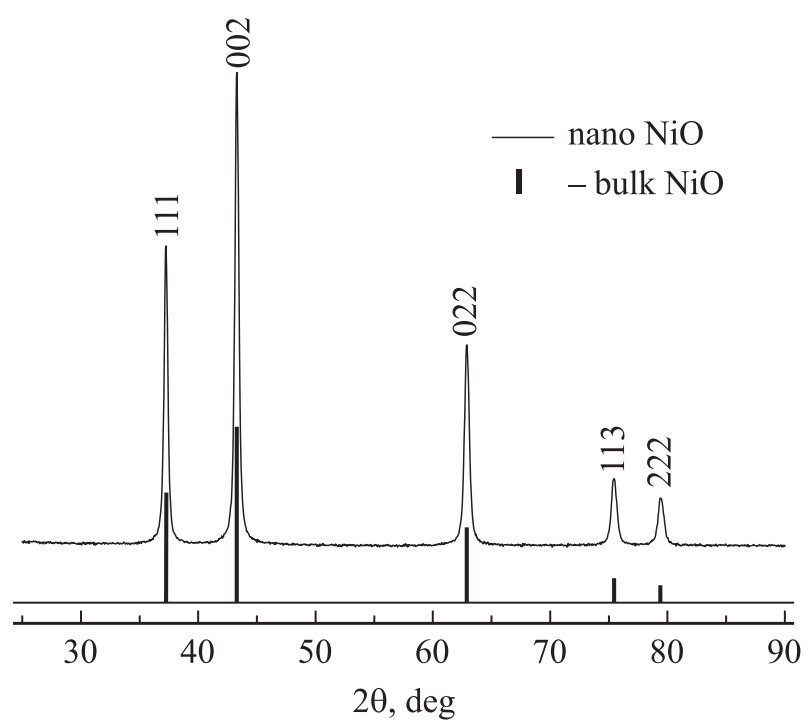

Рис. 1. Экспериментальная дифракционная картина образца nano $\mathrm{NiO}$ в сравнении со штрих-диаграммой, иллюстрирующей положение и относительную интенсивность пиков фазы $\mathrm{NiO}$. 


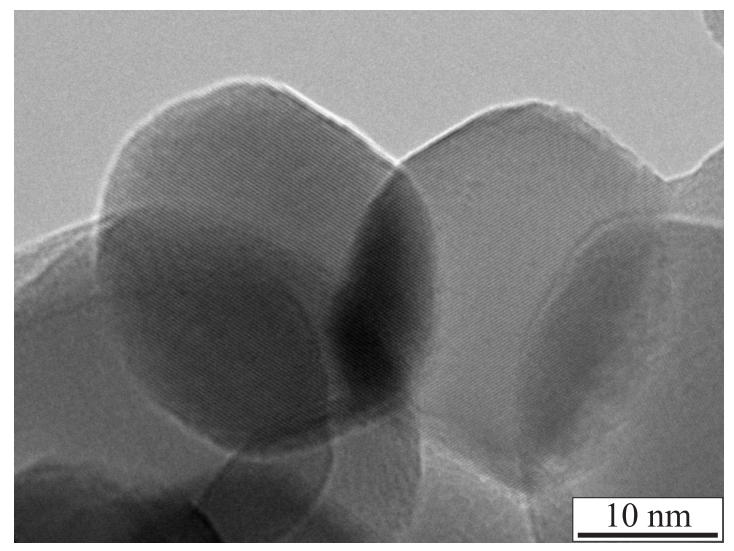

Рис. 2. Результаты просвечивающей электронной микроскопии (НRTEM) образца nano $\mathrm{NiO}$.

микрофотографий) составил $25 \mathrm{~nm}$, что близко к размеру области когерентного рассеяния.

Также для сравнения были исследованы магнитные свойства „объемного“ поликристаллического оксида никеля, который был спечен в таблетку при $600^{\circ} \mathrm{C}$ из реактива NiO. Этот образец обозначен как bulk $\mathrm{NiO}$.

2.2. Изме рения магнитных св ойств. Квазистатические магнитные измерения проводились на вибрационном магнетометре (VSM) [27]. Исследуемый порошок фиксировался в измерительной капсуле в парафине. Для измерений температурных зависимостей магнитного момента $M(T)$ использовались режимы охлаждения без поля (ZFC - zero field cooling) и во внешнем поле (FC - field cooling). Измерения кривых намагничивания $M(H)$ в импульсных магнитных полях проводились на установке, работающей в Институте физики им. Л.В. Киренского СО РАН (Красноярск). Исследуемый порошок в количестве $64 \mathrm{mg}$ надежно фиксировался в индукционном датчике импульсного магнитометра. Длительность импульса составляла $16 \mathrm{~ms}$. Изотермы намагничивания измерялись при температуре $77.4 \mathrm{~K}$ при различных амплитудах импульса магнитного поля вплоть до $250 \mathrm{kOe}$.

\section{3. Результаты и обсуждение}

На рис. 3, $а$ представлены температурные зависимости магнитного момента образца nano $\mathrm{NiO}$ в полях $H=1 \mathrm{kOe}$ (в условиях $\mathrm{ZFC}$ и $\mathrm{FC}$ ), $10 \mathrm{kOe}$ (в условиях $\mathrm{ZFC}$ ) и $H=60 \mathrm{kOe}$ (полученные из изотерм $M(H)$, см. ниже). Данные рис. 3, $a, b$ приведены в единицах emu/g (магнитный момент единицы массы образца), разделенных на величину внешнего поля, $-M(T) / H$. Также на pис. 3, $a$ представлена зависимость $M(T) / H$ для образца bulk $\mathrm{NiO}$ во внешнем поле $1 \mathrm{kOe}$. Для этого образца, как и ожидается для объемных AF-поликристаллических материалов, зависимость $M(H)=\chi_{\mathrm{AF}} H-$ линейная безгистерезисная функция, эффектов термомагнитной предыстории не наблюдается. Следовательно, зависи- мость $M(T) / H$ образца bulk $\mathrm{NiO}$ соответствует температурному поведению АF-восприимчивости объемного поликристаллического оксида никеля $\left(M(T) / H=\chi_{\mathrm{AF}}(T)\right)$, имеющего температуру Нееля $523 \mathrm{~K}$ [28].

Применительно к данным рис. 3, $a$ выражение (2) перепишется в виде

$$
M_{\text {tot }}(T) / H=M_{\mathrm{FM}}(T) / H+\chi_{\mathrm{AF}}(T) .
$$

Из рис. 3, $a$ видно, что наибольшее различие между данными для поликристаллического и наноразмерного $\mathrm{NiO}$ имеет место для $H=1 \mathrm{kOe}$, а при $H=60 \mathrm{kOe}$ зависимость $M(T) / H$ образца nano $\mathrm{NiO}$ практически совпадает с $\chi_{\mathrm{AF}}(T)$ образца bulk NiO. Это объясняется вкладом первого члена выражения (4), который насыщается в больших полях (в насыщении $M_{\mathrm{FM}} \approx$ const и $\left.d M_{\mathrm{FM}}(H) / d H \approx 0\right)$.
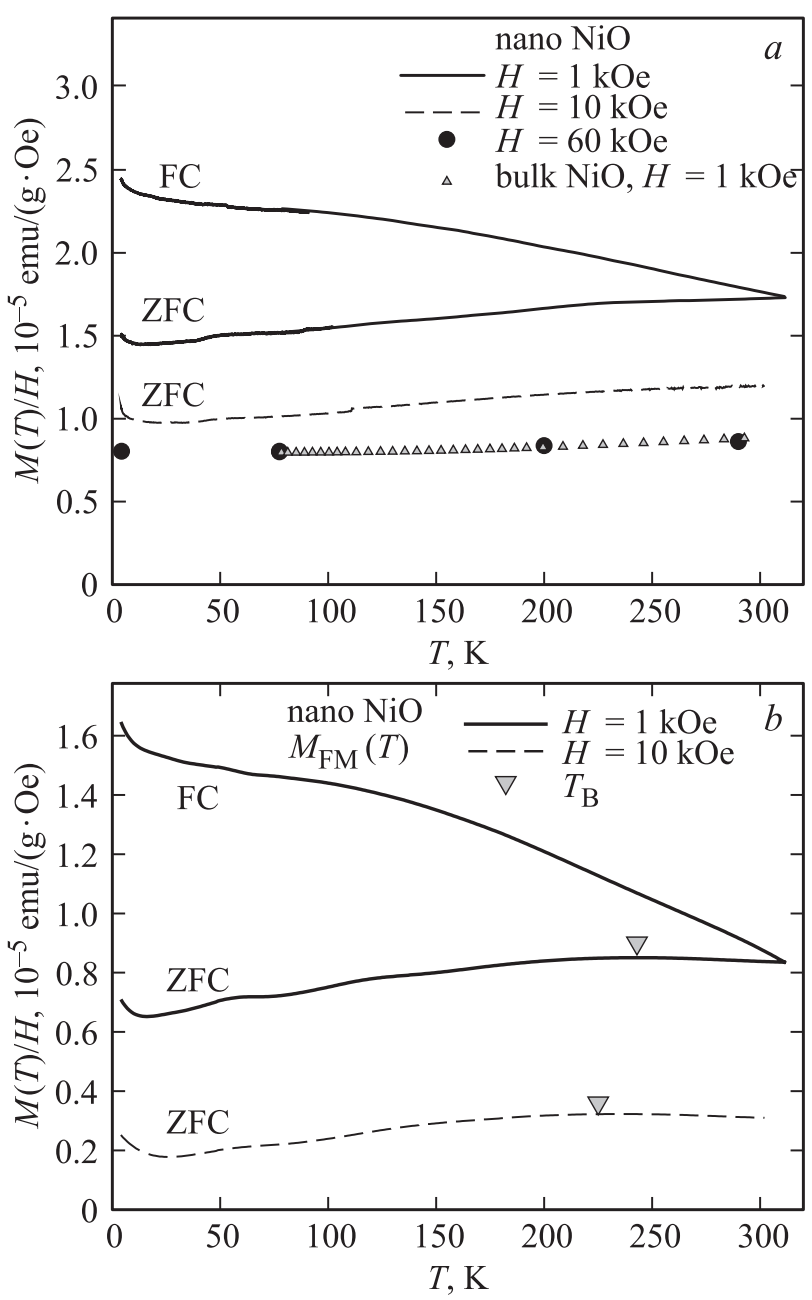

Рис. 3. $a)$ - Температурные зависимости магнитного момента $M(T) / H$ образца nano $\mathrm{NiO}$ во внешних полях $1,10 \mathrm{kOe}$, и значения $M(H=60 \mathrm{kOe}) / 60 \mathrm{kOe}$ при различных температуpax; также приведены данные для образца bulk $\mathrm{NiO}$ в поле $H=1$ kOe. $b)$ - Зависимости $M(T) / H$, соответствующие FMподсистеме образца nano $\mathrm{NiO}$, в полях $1,10 \mathrm{kOe}$ после вычета $\mathrm{AF-составляющей} \mathrm{согласно} \mathrm{выражению} \mathrm{(4);} \mathrm{указана} \mathrm{темпера-}$ тура максимума $\left(T_{B}\right)$ зависимостей $M_{\mathrm{FM}}(T)$ в условиях ZFC. 
Рассмотрим эволюцию зависимостей $M(T)$-образца nano $\mathrm{NiO}$. Видно, что зависимости $M(T)$ в условиях ZFC монотонно возрастают с ростом температуры в диапазоне выше $30 \mathrm{~K}$. Для зависимости $M(T)$ в поле $H=1 \mathrm{kOe}$ имеет место тенденция к выходу на насыщение в области температур около $250 \mathrm{~K}$. Это было бы естественно связать с наличием максимума зависимости $M(T)$ в условиях ZFC, т.е. характерной температурой блокировки $T_{\mathrm{B}}$ суперпарамагнитных частиц. С увеличением внешнего поля температура блокировки должна сдвигаться в область низких температур. Однако из данных для образца nano $\mathrm{NiO}$ этого не следует: напротив, зависимость $M(T)$ при $H=10 \mathrm{kOe}-$ возрастающая функция без признаков насыщения.

С другой стороны, согласно выражению (4), для выделения FM-вклада можно вычесть AF-составляющую. Графики, приведенные на рис. $3, b$, получены путем вычитания экспериментальных данных $\chi_{\mathrm{AF}}(T)$ образца bulk $\mathrm{NiO}$ из соответствующих зависимостей $M(H) / H$ образца nano $\mathrm{NiO}$. Для полученных зависимостей $M_{\mathrm{FM}}(T) / H$, соответствующих FM-подсистеме, наблюдается стандартное для суперпарамагнитных систем поведение: отчетливый максимум температурной зависимости $M_{\mathrm{FM}}(T)$ (в случае ZFC) при температуре блокировки $T_{\mathrm{B}}$ с ростом поля сдвигается в область низких температур $\sim 245 \mathrm{~K}$ при $H=1 \mathrm{kOe}$ и $\sim 225 \mathrm{~K}$ при $H=10 \mathrm{kOe}$.

При величине константы магнитной анизотропии объемного $\mathrm{NiO} \quad K \approx 0.8 \cdot 10^{5} \mathrm{erg} / \mathrm{cm}^{3} \quad[28]$ значение $T_{\mathrm{B}} \approx 245 \mathrm{~K}$, согласно выражению (1), соответствует частицам размера $\sim 23 \mathrm{~nm}$. Это хорошо согласуется с размером частиц образца nano $\mathrm{NiO}$, полученным из данных HRTEM $(\sim 25 \mathrm{~nm})$. Отметим, что указанное значение $T_{\mathrm{B}}$ примерно соответствует среднему размеру частиц, а необратимое поведение зависимостей $M(T)$ и гистерезис $M(H)$ в области температур $T>T_{\mathrm{B}}$ (см. ниже) определяются частицами наибольшего размера ввиду распределения по размерам. Таким образом, температурное поведение АF-восприимчивости „ядра““ наночастиц может существенно влиять на определение их температур блокировки, на что в данной работе указано впервые.

На рис. 4, $a, b$ приведены гистерезисные зависимости $M(H)$ образца nano $\mathrm{NiO}$ при температурах 77 и $290 \mathrm{~K}$ соответственно. Их характер однозначно свидетельствует о сосуществовании двух обсуждающихся магнитных подсистем и суперпозиции магнитных откликов от них в общую кривую намагничивания $M_{\text {tot }}(H)$

$$
M_{\text {tot }}(H)=M_{\mathrm{FM}}(H)+\chi_{\mathrm{AF}} H .
$$

Для FM-подсистемы ожидается выход на насыщение, что должно подтверждаться линейным характером зависимости $M_{\text {tot }}(H)$ в диапазоне достаточно больших магнитных полей. На рис. 5 приведены данные, полученные для образца nano $\mathrm{NiO}$ в импульсном магнитном поле в диапазоне до $250 \mathrm{kOe}$ совместно с данными вибрационного магнетометра VSM в диапазоне до $60 \mathrm{kOe}$. Из рис. 5 видно, что зависимость $M_{\text {tot }}(H)$ линейна по полю в исследуемом диапазоне полей. Следовательно,

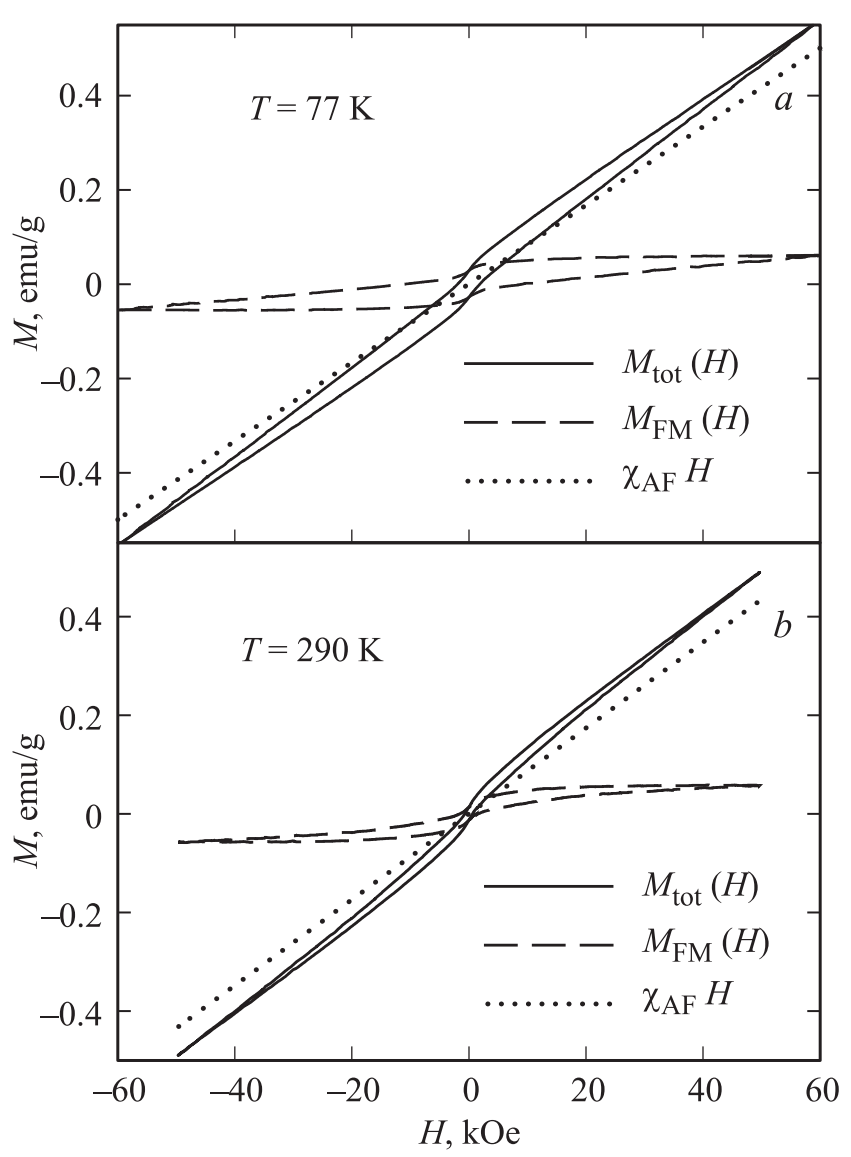

Рис. 4. Гистерезисные зависимости намагничивания $M_{\text {tot }}(H)$ образца nano $\mathrm{NiO}$, прямые линии $\chi_{\mathrm{AF}} H$, соответствующие AFвкладу и полученные из выражения (5) гистерезисные зависимости $M_{\mathrm{FM}}(H)$, соответствующие FM-вкладу, при разных температурах: $a-T=77 \mathrm{~K}, b-T=290 \mathrm{~K}$.

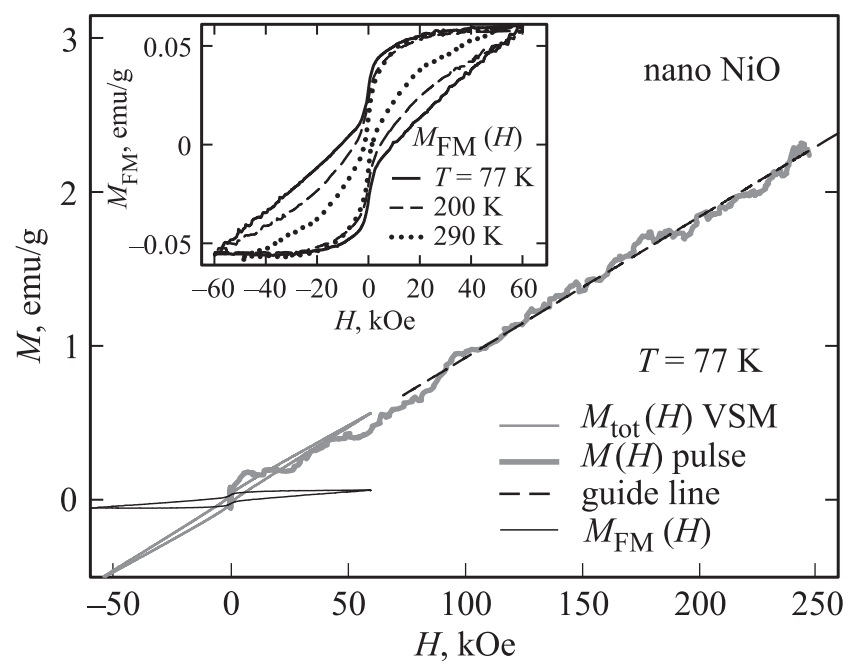

Рис. 5. Кривая намагничивания $M_{\text {tot }}(H)$ образца nano $\mathrm{NiO}$ в импульсном поле до $250 \mathrm{kOe}$ совместно с данными вибрационного магнетометра VSM (до $60 \mathrm{kOe}$ ) при $T=77 \mathrm{~K}$; также показан FM-вклад $M_{\mathrm{FM}}(H)$ в общую кривую намагничивания. На вставке: зависимости $M_{\mathrm{FM}}(H)$ при различных температурах. 
вклад FM-подсистемы, действительно, выходит на насыщение, по крайней мере для температурного диапазона выше $\sim 77 \mathrm{~K}$. Наклон зависимости $M_{\text {tot }}(H)$ в диапазоне полей больше $\sim 100 \mathrm{kOe}$ хорошо согласуется с величиной $\chi_{\mathrm{AF}}$ образца bulk $\mathrm{NiO}$ (рис. 3,a) при температуре измерения $77 \mathrm{~K}$.

Прямые линии на рис. 4, $a, b$ соответствуют вкладу AFподсистемы $\left(\chi_{\mathrm{AF}} H\right)$ при значениях $\chi_{\mathrm{AF}}$, определенных по температурной зависимости $M(T) / H$ образца bulk $\mathrm{NiO}$ (рис. 3,a). Вычитая АF-вклад из полной намагниченности образца, можно получить зависимости $M_{\mathrm{FM}}(H)$ из выражения (5): $M_{\mathrm{FM}}(H)=M_{\mathrm{tot}}(H)-\chi_{\mathrm{AF}} H$. Рис. 4, $a, b$, a также вставка на рис. 5 иллюстрируют эти зависимости. Как видно, зависимости $M_{\mathrm{FM}}(H)$ имеют тенденцию к выходу на насыщение в области полей $\sim 60 \mathrm{kOe}$; коэрцитивная сила уменьшается с ростом температуры.

Величина намагниченности насыщения FM-подсистемы (см. вставку на рис. 5) составляет примерно $\sim 0.06 \mathrm{emu} / \mathrm{g}$. При величине магнитного момента $\mathrm{Ni}^{2+} \approx(2-2.3) \mu_{\mathrm{B}}$ в $\mathrm{NiO}$ [5] это значение соответствует доле нескомпенсированных спинов $\sim 4 \cdot 10^{-4}$ от всех атомов никеля в оксиде. Для частицы размером $\sim 25 \mathrm{~nm}$ количество нескомпенсированных спинов по модельной гипотезе (3) составляет примерно 80 при $b=1 / 3$, что соответствует доле $\sim 1.5 \cdot 10^{-4}$ от всех атомов $\mathrm{Ni}$ в частице такого размера. Для наночастицы размером $25 \mathrm{~nm}$ значение $\mu_{\text {unc }}$ составляет примерно $430 \mu_{\mathrm{B}}$ по экспериментально определенной величине насыщения $M_{\mathrm{FM}}(H)$

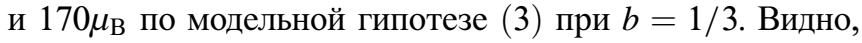
что модельная гипотеза Нееля дает согласие с экспериментом по порядку величины нескомпенсированных магнитных моментов в $\mathrm{NiO}$. Это свидетельствует о том, что FM-вклад вызван дефектами на поверхности частиц.

Отметим, что для температуры $4.2 \mathrm{~K}$ применяемая выше процедура разделения FM- и АF-вкладов не позволила получить зависимость $M_{\mathrm{FM}}(H)$ с выходом на насыщение в полях порядка $60 \mathrm{kOe}$ Укажем две причины, мешающие разделению указанных вкладов. Вопервых, выражение (2) подразумевает, что FM- и AFвклады независимы друг от друга. Однако между AF- и FM-подсистемами может существовать обменная связь, что, в частности, должно приводить к наблюдаемому эффекту обменного смещения петли гистерезиса после охлаждения во внешнем поле. Это наблюдалось для $\mathrm{NiO}[4,29,30]$ и других AF-наночастиц [31-35]. Как правило, при высоких температурах величина обменного смещения уменьшается $[4,12,30]$, что и позволяет рассматривать AF-наночастицу как две независимые подсистемы. Видимо, в области низких температур обменная связь AF- и FМ-подсистем приводит к более медленному насыщению последней с ростом поля. Во-вторых, в магнитоупорядоченных наночастицах практически всегда существуют поверхностные спины, которые обменно не связаны с „внутренними“ спинами [2,12,35-40]. При низких температурах эта дополнительная подсистема демонстрирует „спин-стекольное“ поведение, а при повышении температуры ведет себя как парамагнитная фаза (невзаимодействующие спины). Заметный рост магнитного момента при уменышении температуры в области ниже $30 \mathrm{~K}$, наблюдаемый для образца nano $\mathrm{NiO}$ (см. рис. 3), указывает на присутствие этой дополнительной „спин-стекольной“ подсистемы поверхностных атомов. С ростом температуры вклад этой подсистемы в результирующий магнитный отклик наночастиц становится пренебрежимо мал (уменьшается пропорционально $\propto 1 / T)$, что позволяет непротиворечиво разделить AF- и FM-вклады в наночастицах NiO.

\section{4. Заключение}

На основании исследования магнитных свойств наночастиц $\mathrm{NiO}$ в магнитных полях до $250 \mathrm{kOe}$ продемонстрировано, что для наночастиц размером $\sim 25 \mathrm{~nm}$ магнитный вклад (величина магнитной восприимчивости $\left.\chi_{\mathrm{AF}}\right)$ от AF-упорядоченного „ядра“ с хорошей точностью соответствует поведению объемного антиферромагнитного NiO. Это позволяет разделить AF- и FMвклады для широкой области температур (по крайней мере, выше $\sim 70 \mathrm{~K})$. FМ-вклад возникает вследствие дефектов на поверхности частиц, а величина нескомпенсированного момента согласуется с модельными представлениями, предложенными Неелем. Учет температурного поведения AF-восприимчивости $\chi_{\mathrm{AF}}(T)$,ядра“ частиц необходим для корректного определения температуры суперпарамагнитной блокировки частиц, которая для исследованного образца составляет $\sim 245 \mathrm{~K}$ в поле $H=1 \mathrm{kOe}$. При низких температурах проявляется вклад еще одной магнитной подсистемы - части поверхностных спинов, обменно не связанных с AF-,ядром“.

\section{Список литературы}

[1] S. Mørup, D.E. Madsen, C. Fradsen, C.R.H. Bahl, M.F. Hansen. J. Phys.: Condens. Matter 19, 213202 (2007).

[2] R.H. Kodama, A.E. Berkowitz. Phys. Rev. B 59, 6321 (1999).

[3] Yu.L. Raikher, V.I. Stepanov. J. Phys.: Condens. Matter. 20, 204120 (2008).

[4] S.A. Makhlouf, F.T. Parker, F.E. Spada, A.E. Berkowitz. J. Appl. Phys. 81, 8, 5561 (1997).

[5] S.D. Tiwari, K.P. Rajeev. Solid State Commun. 152, 1080 (2012).

[6] S.A. Makhlouf, F.T. Parker, A.E. Berkowitz. Phys. Rev. B 55, R14717 (1997).

[7] D.A. Balaev, A.A. Krasikov, A.A. Dubrovskiy, S.I. Popkov, S.V. Stolyar, R.S. Iskhakov, V.P. Ladygina, R.N. Yaroslavtsev. J. Appl. Phys. 120, 183903 (2016).

[8] A.A. Lepeshev, I. V. Karpov, A.V. Ushakov, D. A. Balaev, A.A. Krasikov, A.A. Dubrovskiy, D.A. Velikanov, M.I. Petrov. J. Supercond. Nov. Magn. (2016). DOI 10.1007/s10948-0163885-4

[9] Ю.А. Кумзеров, Н.Ф. Картенко, Л.С. Парфеньева, И.А. Смирнов, А.А. Сысоева, Н. Misiorek, А. Jezowski. ФTT 54, 5, 1000 (2012.)

[10] D.A. Balaev, A.A. Krasikov, A.A. Dubrovskiy, S.I. Popkov, S.V. Stolyar, O.A. Bayukov, R.S. Iskhakov, V.P. Ladygina, R.N. Yaroslavtsev. J. Magn. Magn. Mater. 410, 71 (2016). 
[11] Д.А. Балаев, А.А. Красиков, А.А. Дубровский, С.В. Семёнов, О.А. Баюков, С.В. Столяр, Р.С. Исхаков, В.П. Ладыгина, Л.А. Ищенко. ЖЭТФ 146, 546 (2014).

[12] A. Punnoose, H. Magnone, M.S. Seehra, J. Bonevich. Phys. Rev. B 64, 174420 (2001).

[13] Q.A. Pankhurst, N.T.K. Thanh, S.K. Jones, J. Dobson. J. Phys. D 42, 224001 (2009).

[14] Q.A. Pankhurst, J. Connolly, S.K. Jones, J. Dobson. J. Phys. D 36, R167 (2003).

[15] K. Dobretsov, S. Stolyar, A. Lopatin. Acta Otorhinolaryngol Ital. 35, 2, 97 (2015).

[16] L. Néel, C.R. Acad. Sci. Paris 253, 1286 (1961).

[17] L. Néel, C.R. Acad. Sci. Paris 253, 203 (1961).

[18] L. Néel, C.R. Acad. Sci. Paris 252, 4075 (1961).

[19] N.J.O. Silva, A. Millan, F. Palacio, E. Kampert, U. Zeitler, V.S. Amaral. Phys. Rev. B 79, 104405 (2009).

[20] M.S. Seehra, V.S. Babu, A. Manivannan, J.W. Lynn. Phys. Rev. B 61, 3513 (2000).

[21] A. Punnoose, T. Phanthavady, M.S. Seehra, N. Shah, G.P. Huffman. Phys. Rev. B 69, 054425 (2004).

[22] N.J.O. Silva, V.S. Amaral, L.D. Carlos. Phys. Rev. B 71, 184408 (2005).

[23] M.S. Seehra, V. Singh, X. Song, S. Bali, E.M. Eyring. J. Phys. Chem. Solids 71, 1362 (2010).

[24] R.P. Guertin, N. Harrison, Z.X. Zhou, S. McCall, F. Drymiotis. J. Magn. Magn. Mater. 308, 97 (2007).

[25] C. Gilles, P. Bonville, H. Rakoto, J.M. Broto, K.K.W. Wong, S. Mann. J. Magn. Magn. Mater. 241, 430 (2002).

[26] V. Markovich, R. Puzniak, Y. Skourski, A. Wisniewski, D. Mogilyanski, G. Jung, G. Gorodetsky. J. Phys.: Condens. Matter 24, 266001 (2012).

[27] А.Д. Балаев, Ю.В. Бояршинов, М.М. Карпенко, Б.П. Хрусталев. ПТЭ 3, 167 (1985).

[28] M. Tadic, D. Nikolic, M. Panjan, G.R. Blake. J. Alloys Comp. 647, 1061 (2015).

[29] S.A. Makhlouf, H. Al-Attar, R.H. Kodama. Solid State Commun. 145, 1 (2008).

[30] M.S. Seehra, A. Punnoose. Solid State Commun. 128, 299 (2003).

[31] A. Punnoose, M.S. Seehra. J. Appl. Phys. 91, 10, 7766 (2002).

[32] C. Diaz-Guerra, M. Vila, J. Piqueras. Appl. Phys. Lett. 96, 193105 (2010).

[33] Д.А. Балаев, А.А. Красиков, А.А. Дубровский, С.В. Семёнов, С.И. Попков, С.В. Столяр, Р.С. Исхаков, В.П. Ладыгина, Р.Н. Ярославцев. ФТТ 58, 2, 280 (2016).

[34] N.J.O. Silva, V.S. Amaral, A. Urtizberea, R. Bustamante, A. Millán, F. Palacio, E. Kampert and U. Zeitler, S. de Brion, O. Iglesias, A. Labarta. Phys. Rev. B 84, 104427 (2011).

[35] S. Giri, M. Patra, S. Majumdar. J. Phys.: Condens. Matter 23, 073201 (2011).

[36] V.L. Kirillov, D.A. Balaev, S.V. Semenov, K.A. Shaikhutdinov, O.N. Martyanov. Mater. Chem. Phys. 145, 75 (2014).

[37] Yu.A. Koksharov, S.P. Gubin, I.D. Kosobudsky, G.Yu. Yurkov, D.A. Pankratov, L.A. Ponomarenko, M.G. Mikheev, M. Beltran, Y. Khodorkovsky, A.M. Tishin. Phys. Rev. B 63, 012407 (2000).

[38] D.A. Balaev, A.A. Dubrovskiy, K.A. Shaykhutdinov, O.A. Bayukov, S.S. Yakushkin, G.A. Bukhtiyarova, O.N. Martyanov. J. Appl. Phys. 114, 163911 (2013).

[39] A.A. Dubrovskiy, D.A. Balaev, K.A. Shaykhutdinov, O.A. Bayukov, O.N. Pletnev, S.S. Yakushkin, G.A. Bukhtiyarova, O.N. Martyanov. J. Appl. Phys. 118, 213901 (2015).

[40] K. Nadeem, H. Krenn, T. Traussnig, R. Würschum, D.V. Szabo, I. Letofsky-Papst. J. Appl. Phys. 111, 113911 (2012). 